\title{
Using wearable electronic sensors for assessing contacts between individuals in various environments
}

\author{
N Voirin ${ }^{*}$, J Stehlé 2 , A Barrat ${ }^{2}$, C Cattuto ${ }^{3}$, L Isella ${ }^{3}$, J-F Pinton ${ }^{4}$, M Quaggiotto ${ }^{3}$, N Khanafer ${ }^{1}$, W Van den Broeck ${ }^{3}$,

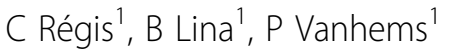

From International Conference on Prevention \& Infection Control (ICPIC 2011)

Geneva, Switzerland. 29 June - 2 July 2011

\section{Introduction / objectives}

Transmission of hospital acquired infections (HAI) is mainly based on contacts between patients, patients and health care workers (HCWs) and between HCWs. Description and quantification of contacts at hospital are key information for HAIs epidemiology and implementing control measures.

\section{Methods}

The SocioPatterns project (http://www.sociopatterns.org) has developed an technology based on RFID badges that provides a reliable infrastructure to detect face-to-face proximity of individuals. The system was tested at a scientific conference, in a primary school and in a hospital unit.

\section{Results}

At the scientific conference, 26,040 contacts (average duration of 54 seconds) were recorded among 402 participants during two days. At school, 77,226 contacts (average number of 160 contacts per children per day) were observed among 233 children during two days. In the geriatric unit, 50 staff and 29 patients participated to the study during 5 consecutive days. Statistical analyses are ongoing. For each study, time-resolved datasets on contact patterns were generated. Analyses provided frequency and duration of contacts, and contact matrices. Modeling of the spread of infections will be presented.

\section{Conclusion}

The study of contacts networks is important to better address the prevention and control of known and emerging HAIs. They are also useful tools to build better models for decision making in public health. Collecting contacts data in the hospital setting, using such electronic devices, appeared to be appropriate and will be an added value to other approaches such as observational audit.

\section{Disclosure of interest \\ None declared.}

\section{Author details}

${ }^{1}$ Hospices Civils de Lyon - Université Lyon 1 - CNRS, Lyon, France. ${ }^{2}$ CNRS ISI Foundation, Marseille, France. ${ }^{3}$ ISI Foundation, Torino, Italy. ${ }^{4}$ ENS - CNRS, Lyon, France.

Published: 29 June 2011

\section{doi:10.1186/1753-6561-5-S6-051}

Cite this article as: Voirin et al.: Using wearable electronic sensors for assessing contacts between individuals in various environments. BMC Proceedings 2011 5(Suppl 6):O51. 